\title{
Lesioni iatrogene del nervo accessorio spinale: responsabilità professionale, rischio del medico e aspetti giuridici
}

\author{
Antonio Frailis ${ }^{1}$
}

1 Direttore Servizio Igiene e Sanità pubblica AUSL, Sanluri (CA). Consulente medico-legale della Procura e del Tribunale ordinario di Cagliari

\begin{abstract}
The majority of lesions of the spinal accessory nerve occur as an iatrogenic injury after lymph node biopsy in the posterior cervical triangle. In most cases the accessory nerve palsy is not recognised immediately after the injury. Therefore surgical repair is often performed too late to regain sufficient function of the paralytic trapezium muscle.

We report a case of a 23 year old patient who was taken in Hospital for a non Hodgkin's lymphoma. After biopsy a strong pain to right shoulder emerged. Despite the man's complaints of left arm weakness the doctor waited several weeks to look into the matter. Only one year later he was properly diagnosed with a iatrogenic accessory nerve injury.

The iatrogenic injuries of the accessory nerve may lead to significant lifelong disability. The diagnosis is not always made in time with consequent delay in repair may be regarded as an unfavourable issue during medical-legal discussions. The Author recommends in interest to prevent nerve injury in the posterior triangle of the neck to perform operation in special department.
\end{abstract}

Keywords: spinal accessory nerve, lymph node biopsy, professional liability

Iatrogenic injury of the spinal accessory nerve. Professional liability, medical risk and juridical aspects. Pratica Medica \& Aspetti Legali 2007; 1(1): 33-42

\section{INTRODUZIONE}

La lesione del nervo accessorio è la complicazione più frequente fra le procedure chirurgiche nel triangolo posteriore del collo. I sintomi prodotti dalla paralisi del trapezio sono invalidanti. Le componenti dell'invalidità sono dolore, limitazione dell'abduzione e abbassamento della spalla colpita. Una conoscenza dettagliata del decorso del nervo e le sue relazioni anatomiche sono essenziali per evitare lesioni. Utili punti di riferimento anatomici sono la vena giugulare interna prossimale nel triangolo anteriore e il punto di Erb nel triangolo posteriore. La prevenzione della lesione del nervo accessorio è la miglior gestione. Le indicazioni per le biopsie del linfonodo del collo dovrebbero essere sempre attentamente vagliate. L'uso dell'anestetico generale senza paralisi è raccomandato in caso in cui sia necessaria una biopsia da escissione. È essenziale un'adeguata esposi- zione. Qualora il nervo necessiti di essere reso visibile deve sempre essere individuato e richiedere un'attenta analisi. Un nervo diviso o danneggiato è curato meglio con una riparazione primaria entro 3 mesi dalla lesione.

La lesione del nervo accessorio è segnalata come la più frequente complicazione delle procedure chirurgiche del triangolo del collo. Considerando un ampio numero di biopsie cervicali del linfonodo eseguite, si tratta di un evento relativamente poco frequente. La disfunzione del nervo accessorio può complicare una varietà di lesioni del nervo e viene riportata come conseguenza della cannulazione della vena giugulare interna e della tiroidectomia. L'importanza di questa lesione è il risultato di una disabilità causata dalla perdita della funzione del trapezio. Una lesione accidentale del nervo accessorio nel corso di una biopsia del linfonodo non è accettabile da un punto di vista medico-legale. Una sindrome della spalla a cui segue una dissezione radicale del collo, attribuita ad una 
paralisi del trapezio dopo la distruzione del nervo accessorio, fu descritta da Nahum nel 1961. I principali sintomi della sindrome erano:

- dolore nella regione dell'articolazione della spalla non meglio localizzata, né riprodotta dalla pressione in alcun punto particolare, né dal movimento in alcuna direzione; si poteva verificare a riposo e aggravava con qualsiasi movimento dell'articolazione della spalla;

- limitazione dell'abduzione dell'articolazione della spalla; veniva mantenuta una gamma pienamente passiva del movimento;

- anomalie anatomiche consistenti nell'abbassamento della spalla colpita, prominenza della scapola e prominenza delle altre articolazioni del muscolo della spalla, il romboide in particolare; non si evidenziano anomalie radiografiche.

La limitazione dell'abduzione può danneggiare seriamente le funzioni della spalla. Ewing e Martin hanno concluso da uno studio su 100 pazienti sottoposti a una dissezione radicale del collo che la disabilità più comune era rappresentata dalla difficoltà nell'uso delle braccia nelle attività che implicano l'uso delle mani sopra il livello della spalla [1]. Le componenti comuni in tutti i casi di lesione del nervo accessorio seguenti a una biopsia cervicale del linfonodo sono date dal fatto che la lesione frequentemente si verificava durante la biopsia di un piccolo linfonodo, detto "shotty", e quando veniva praticata l'anestesia locale l'incisione tendeva ad essere piccola o "endoscopica". Ad un certo punto dell'operazione la maggior parte dei pazienti sentiva un improvviso dolore acuto che si irradiava (diffondeva) sulla spalla o nella regione del collo. Subito dopo l'operazione si riscontrava generale debolezza e fastidio del legamento della spalla e dell'area del collo, lamentele viste come una naturale conseguenza della operazione. Come conseguenza del fastidio, veniva limitato l'uso dell'estremità vicino al legamento della spalla, e ciò era generalmente attribuito al dolore dell'incisione nel post-operatorio. Dopo diversi giorni, si notavano diversi gradi di pesantezza della spalla, e veniva meno l'abduzione del braccio sopra i 90 gradi. Ai pazienti veniva detto generalmente che i problemi sarebbero scomparsi nel tempo. Dopo diverse settimane, o anche dopo diversi mesi, si notava l'allungamento del muscolo laterale del collo con un risultato di un cambiamento del profilo e abbassamento della spalla compromessa.

\section{DESCRIZIONE DEL CASO}

A.M., maschio di 23 anni di razza caucasica, atleta appassionato di canottaggio e tennis, in data 27 aprile 2003, a causa di una persistente febbre elevata accompagnata da tumefazione linfoghian- dolari laterocervicale bilaterale, viene ricoverato presso un Ospedale ad alta specializzazione di Cagliari, dal quale viene subito dimesso e trasferito presso l'Ospedale Oncologico di Cagliari con diagnosi di «Linfoma maligno non Hodgkin anaplastico a grandi cellule».

Durante il ricovero esegue varie indagini strumentali, fra le quali due biopsie linfoghiandolari laterocervicali, in data 28 aprile (la destra) e 12 maggio 2003 (la sinistra).

Subito dopo la prima biopsia inizia ad accusare parestesie dolorose alla spalla destra, impotenza funzionale ingravescente dell'arto superiore destro e una debolezza della rotazione del capo verso sinistra. Preoccupato, pone a conoscenza di questa sintomatologia i Sanitari interessati ed in particolare il chirurgo che ha effettuato la biopsia laterocervicale destra, ricevendo da questo ampia assicurazione sulla pronta scomparsa di questi, a loro detta, "normali ed attesi" sintomi postumi di una biopsia.

Il quadro, però, va peggiorando, tanto che al quadro suddescritto si aggiunge una vistosa debolezza dell'innalzamento della spalla destra con "caduta" di essa.

Insospettito dalle varie giustificazioni fornite di volta in volta dai Sanitari responsabili e dopo aver concluso l'iter terapeutico per il suo male più importante presso l'Ospedale Oncologico, il paziente si rivolge a molti specialisti ortopedici, che prescrivono ogni volta della fisio kinesi terapia (FKT) con riabilitazione della spalla, senza sospettare quella che in seguito si sarebbe palesata come patologia ben più seria.

Si arriva, così, al giugno 2004, quando una elettromiografia mette in evidenza una «lesione totale (sezione completa) del nervo accessorio spinale destro".

Tale lesione spiega totalmente il quadro descritto.

\section{GLI ASPETTI CLINICI}

\section{ANAMNESI ED ESAME OBIETTIVO}

I dati anamnestici e quelli derivanti dall'esame obiettivo del paziente sono riportati di seguito.

Soggetto normotipo in ottime condizioni generali. Cute di colorito roseo, trofica ed elastica. Facies composita. Masse muscolari tonico-trofiche. Mucose visibili rosee e ben idratate. Altezza $178 \mathrm{~cm}$; peso $76 \mathrm{~kg}$; PA 125/90 mmHg.

Pannicolo adiposo sottocutaneo normopresente per sesso ed età.

Capo mesaticefalo, non dolente, né dolorabile nei movimenti di lateralità e flesso-estensione. Abnorme difficoltà a rivolgere il capo a sinistra per lesione funzionale del muscolo sternocleidoma- 
stoideo. Bulbi oculari in sede, normoprotrusi, simmetrici. Pupille isocoriche e isocicliche, normoreagenti alla luce e alla accomodazione. Punti sinusali, frontali e mascellari, non dolenti.

Torace tronco-conico con emitoraci simmetrici nella statica e nella dinamica, simmetricamente normoespansibili. Non bozze, né rientramenti patologici. Suono chiaro polmonare. Si apprezza il murmure vescicolare su tutto l'ambito. Sul dorso si nota un vistoso abbassamento del moncone della spalla destra, un basculamento in fuori della scapola omolaterale con grave impotenza funzionale nell'innalzamento della spalla destra.

Apparato CVS itto visibile e palpabile al $5^{\circ}$ spazio intercostale sinistro sulla linea di repere emiclaveare omolaterale. Aia cardiaca percussoriamente nei limiti. Alla auscultazione i toni sono ritmici, netti, normofrequenti, le pause libere.

Addome piano, CO normointroflessa. Cicatrice xifo-ombelico-pubica per pregressa splenectomia. Non dolente alla palpazione superficiale e profonda lungo il decorso del grosso intestino. Normale timpanismo enterocolico. Peristalsi intestinale presente e valida.

Fegato: la cupola si trova al $5^{\circ}$ spazio intercostale destro sulla linea di repere mammillare omolaterale. Il margine inferiore non si apprezza. Manovra di Murphy negativa.

Milza: splenectomizzato.

Apparato urogenitale: manovra di Giordano negativa bilateralmente. Punti reno-ureterali non dolenti. Genitali esterni come di norma.

Apparato osteoarticolare e motorio: la deambulazione avviene come di norma e non deve essere sostenuta. Le grosse articolazioni sono di forma e volume normali ed i movimenti attivi e passivi avvengono senza dolore e senza limitazione funzionale.

Psiche e sistema nervoso: il paziente è sveglio, vigile e perfettamente in contatto. Le memorie, recente e di fissazione, sono perfettamente conservate. Non sono presenti segni di deficit a focolaio, né a carico dei nervi periferici e/o cranici (eccezion fatta per l' $11^{\circ}$ paio). L'eloquio, spontaneo e indotto, è fluido e sempre correttamente indirizzato.

Romberg negativo. Non slivellamento in Mingazzini. Le prove di coordinazione motoria sono negative per lesione e vengono effettuate con precisione.

Si osservano gli esiti, irreversibili, della lesione totale del nervo accessorio spinale destro e consistenti in: "debolezza della rotazione del capo verso sinistra per paralisi del muscolo sternocleidomastoideo destro, vistoso abbassamento del moncone della spalla destra, un basculamento in fuori della scapola destra, una grave impotenza funzionale nell'innalzamento della spalla destra, il tutto per paralisi della parte superiore del muscolo trapezio".

\section{Anamnesi}

Un'anamnesi attenta è essenziale per evidenziare gli elementi diagnostici più importanti.

L'anamnesi deve valutare le patologie remote, pregressa radioterapia, l'uso di alcol e tabacco, ecc.; deve inoltre comprendere:

- accurato esame fisico;

- età del paziente;

- tempo di insorgenza della massa;

- eventuale crescita o decremento della massa, tempi e modalità;

- iperestesia cutanea e/o dolore spontaneo o provocato;

- febbre;

- intercorrenti problemi dentari;

- pregresso trattamento di lesioni cutanee;

- pregresso tumore del tratto aero-digestivo superiore;

- pregressa tubercolosi (scrofola), sarcoidosi, actinomicosi, infezioni micotiche;

- sintomi di ostruzioni nasale (difficoltà respiratoria);

- eventuale sanguinamento;

- comparsa di otalgia, odinofagia, disfagia, raucedine.

\section{Osservazione}

Losservazione va condotta con il paziente in posizione seduta e testa allo stesso livello dell'esaminatore. Cuoio capelluto, cute della faccia e collo saranno attentamente esaminate (eventuali pigmentazioni cutanee o ulcerazioni). La deviazione di uno dei bulbi oculari, o la perdita di sensibilità della cute della guancia, possono essere dovute ad invasione tumorale della cavità nasale o dei seni paranasali, esplorazione della cavità orale e delle vie respiratorie (orofaringe, faringe). Paziente rilassato; sguardo sull'esaminatore; respirazione libera. Utile un esame endoscopico a fibre ottiche. In assenza di lesioni visibili, la palpazione bimanuale può permettere di scoprire un tumore alla base della lingua ricoperta da mucosa normale, oppure una lesione cancerosa in una cripta tonsillare.

Dopo l'esame della via respiratoria superiore il collo è più efficacemente esaminato.

Un completo esame fisico include anche lo studio di altri apparati: torace, addome, regione pelvica ed estremità. Particolare attenzione alle maggiori stazioni linfonodali: ascella, regione inguinale, ecc. L'esame della regione pelvica deve includere anche l'apparato genitale, retto e vagina nelle donne, retto e prostata nell'uomo.

\section{Stazioni linfonodali della regione del collo}

La diagnostica delle masse del collo si basa su una completa conoscenza dei punti di repere anatomi- 
ci del collo e delle varie stazioni linfonodali che normalmente in esso si trovano e che, nel corso di patologie neoplastiche e/o infiammatorie, possono essere palpate.

Le cinque stazioni linfonodali principali della regione del collo sono:

- I livello: linfonodi sottomentonieri;

- II livello: del III superiore del muscolo sternocleidomastoideo;

- III livello: linfonodi del III medio del muscolo sternocleidomastoideo;

- IV livello: dei linfonodi del III inferiore del muscolo sternocleidomastoideo;

- V livello: linfonodi occipitali, dei processi trasversi delle vertebre cervicali, postero-auricolari, della fossa sopraclavicolare.

Quando si esamina questa regione bisogna essere consapevoli delle varianti anatomiche possibili per non incorrere in errori di valutazione, palpando strutture che possono essere considerate patologiche e che patologiche non sono.

\section{Diagnosi differenziale delle neoplasie della regione del collo}

Da uno studio eseguito su 300 pazienti e della durata di dieci anni, condotto nell'Istituto di Clinica Chirurgica dell'Università di Palermo, è stato rilevato che la diagnosi delle neoplasie della regione del collo ha le seguenti caratteristiche: alterazioni congenite, alterazioni infiammatorie, neoplasie di natura benigna, neoplasia di natura maligna; e le seguenti varianti anatomiche: osso joide, bulbo carotideo, processo stiloide, processo trasverso della I vertebra cervicale [2].

Le alterazioni di natura congenita sono: malformazioni vascolari, cisti del dotto tireoglosso, cisti del dotto brachiale, teratoma, linfoangioma, laringocele.

I processi infiammatori sono: linfoadenite acuta non suppurativa, mononucleosi infettiva, febbre da graffio di gatto, linfoadenite cronica non specifica, AIDS, linfoadenite tubercolare, linfoadenite micotica, cisti sebacea suppurata.

Le patologie benigne: bulbo carotideo aterosclerotico, paraganglioma del corpo della tiroide, cisti congenite (particolarmente comuni in questa regione), mediane (dotto tireoglosso), laterali (residui branchiali).

I tumori espansivi del collo possono invadere la biforcazione carotidea e spostarla lateralmente.

Per differenziarlo da un linfonodo ingrandito, basterà tentare di muovere la massa in senso cefalo-caudale. I tumori del corpo carotideo non potranno muoversi in questa direzione poiché fissati tenacemente al bulbo, mentre il linfonodo potrà essere mosso.

Neoplasie acquisite di natura benigna: noduli tiroidei e gozzo, tumori ghiandolari salivari, paraganglioma del corpo della carotide, tumori dei tessuti molli (lipoma, neuroma, fibroma).

Tumori primari: ghiandole salivari, tiroidei, sarcomi dei tessuti molli, tumori cutanei (melanoma, carcinoma a cellule basali e squamose), tumori del tratto aereo-digestivo superiore, linfoma di Hodgkin's e non Hodgkin's

Tumori metastatici: cute, ghiandole salivari, tiroide, mammella, tratto gastrointestinale e genitourinario, polmone.

La corretta metodica di esecuzione della palpazione dei livelli linfonodali deve avvenire come segue:

- I livello: da questa posizione può essere identificata la ghiandola sottomandibolare che appare di consistenza gommosa e lobulata. I linfonodi di questa area sono normalmente anteriori alla ghiandola sottomandibolare, possono essere meglio evidenziati con la palpazione bimanuale tra sinfisi e angolo della mandibola. I linfonodi di dimensioni leggermente al di sotto di $1 \mathrm{~cm}$ possono essere palpati. La ghiandola parotide e i linfonodi periparatiroidei possono ancora essere esaminati allo stesso tempo;

\begin{tabular}{|cl|}
\hline Livello & \\
\hline I livello & Interessato da tumori di labbra, cavità orale, cute della faccia \\
\hline II livello & Tumori di cavità orale, orofaringe, naso-faringe, ipofaringe, laringe \\
\hline III livello & Interessati in caso di tumore di cavo orale, orofaringe, ipofaringe, laringe e tiroide \\
\hline IV livello & $\begin{array}{l}\text { Interessati da tumori del tratto digestivo superiore (orofaringe, ipofaringe, esofago } \\
\text { cervicale), laringe, ghiandola tiroide, o da tumore a sede sottoclavicolare (linfonodi di }\end{array}$ \\
\hline V livello & $\begin{array}{l}\text { Si distinguono le seguenti stazioni linfonodali: } \\
\text { - linfonodi occipitali e postero-auricolari interessati da tumori della regione posteriore del } \\
\text { cuoio capelluto, orecchio, polmone, tratto gastrointestinale e tratto genito-urinario } \\
\text { - linfonodi para-cervicali: interessati da tumori di naso-laringe, cuoio capelluto regione } \\
\text { posteriore, tiroide } \\
\text { - linfonodi paratiroidei: interessati da tumori del cuoio capelluto, regione frontale e } \\
\text { laterale, naso, guancia }\end{array}$ \\
\hline
\end{tabular}

Tabella I. Interessamento dei livelli linfonodali secondo la sede del tumore primario 
- II livello: i linfonodi di II livello sono meglio palpati dal posizionamento del pollice dell'esaminatore al III superiore del muscolo sternocleidomastoideo quasi a pinzare il muscolo stesso, la testa deve essere leggermente inclinata dal lato dell'osservatore.

In Tabella I è riportato l'nteressamento dei livelli linfonodali secondo la sede del tumore primario.

\section{METODI DIAGNOSTICI}

\section{Fine-needle aspiration biopsy (FNAB)}

È una tecnica ormai standardizzata che permette di aspirare le cellule della massa tumorale per eseguire l'esame citologico.

Le Fevbre et al. sono arrivati alla conclusione che con un'accurata anamnesi, un buon esame obiettivo ed eseguendo la FNAB della lesione, si raggiunge una accuratezza diagnostica del 91\%. Nei restanti casi in cui non si riesce a porre una diagnosi certa si esegue la biopsia incisionale ed escissionale [3].

\section{Biopsia incisionale ed escissionale}

Una FNAB che lascia dubbi diagnostici è un esame incompleto. A questo punto è necessario eseguire la biopsia incisionale ed escissionale:

- per larghe masse, nell'impossibilità di asportare per intero la formazione, si preferisce eseguire la biopsia incisionale a cielo aperto;

- nella biopsia incisionale viene asportata soltanto una piccola porzione della massa;

- se la massa è di piccole dimensioni (es. linfonodo) essa può essere asportata nella sua interezza con una piccola incisione effettuata al di sopra di essa (biopsia escissionale).

Per l'esecuzione il chirurgo dovrà scegliere la massa (fra le tante che si presentano) che più si presta a tale scopo (ad es. tra i linfonodi il più idoneo). Non sempre il linfonodo più grosso è il più facile da prelevare e non è sempre il più idoneo per l'esame istologico; le masse più larghe contengono al loro interno tessuto necrotico e vanno evitate poiché non offrono una cellularità di qualità idonea per l'esame. Se, ad esempio, si è in presenza di un linfoma con interessamento dei linfonodi del collo, quelli più piccoli (diametro $<2 \mathrm{~cm}$ ), per l'architettura del linfonodo stesso e per il buon contenuto cellulare, consentono di porre ottimamente la diagnosi. Se si sospetta un'infezione sul pezzo asportato, è bene eseguire esame colturale al fine di mettere in evidenza un'eventuale crescita batterica e/o micotica.

\section{Immagine radiografica}

La biopsia permette di porre una diagnosi istologica. Lindagine radiologica, comunque, assume grande rilevanza, sia dal punto di vista diagnostico che di staging.

Tutti i pazienti con una diagnosi di cancro devono essere sottoposti a RX del torace standard. In caso di tumori primari della base del collo, nell' $80 \%$ dei casi si noterà già all'RX un'immagine in regione sopraclavicolare.

Nell'uomo la maggior parte di questi tumori sono a partenza delle alte vie aeree e dal tratto digestivo superiore. È utile eseguire ancora altre indagini radiologiche, ad esempio TAC della testa, del collo, del torace, addome superiore e inferiore con o senza MDC, al fine di identificare il sito primario ed evidenziare eventuali metastasi.

Linfonodi di piccole dimensioni e non palpabili possono essere sede di metastasi da carcinoma: la loro comparsa è utile ai fini della stadiazione della malattia e soprattutto ai fini diagnostici.

Le immagini radiografiche di pazienti con adenocarcinoma metastatico, con carcinoma scarsamente differenziato e con carcinoma neuroendocrino sono differenti.

Posta la diagnosi, alcuni pazienti potranno essere sottoposti a un trattamento chirurgico e/o chemioterapico radicale, oppure solo palliativo.

\section{Endoscopia}

Quando l'anamnesi, l'esame obiettivo e le immagini radiologiche suggeriscono la presenza di un cancro primario del tratto aereo-digestivo superiore, diventa utile eseguire, in anestesia generale, una naso-faringoscopia, una laringoscopia diretta, una esofagoscopia e una broncoscopia.

Deve essere anche eseguito un attento esame della faccia laringea dell'epiglottide, dell'ipofaringe, della base della lingua e delle tonsille (per la possibilità di un carcinoma a partenza dalle cripte tonsillari).

L'esame endoscopico, oltre che dare una immagine diretta della lesione, permette anche di eseguire dei prelievi bioptici o un brashing. Sono da bandire le cosiddette "biopsie cieche" eseguite senza visione diretta della lesione.

\section{CONCLUSIONI}

Un'accurata programmazione diagnostica e terapeutica delle masse del collo è fondamentale per un auspicabile miglioramento dei risultati di tali patologie che nelle forme maligne presentano a tutt'oggi ancora una prognosi infausta, potendo essere la manifestazione di neoplasie loco regionali. Il trattamento dei carcinomi della testa e del collo si avvale, nelle diverse manifestazioni cliniche, di chirurgia, radioterapia e chemioterapia secondo protocolli unimodali o di associazione. In ogni caso questo comporta che la strategia terapeutica di questi tumori necessiti oggi di un approccio multidisciplinare da effettuarsi preferibil- 
mente in centri dotati delle diverse competenze specialistiche.

\section{La terapia chirurgica e i suoi principi}

La rimozione per intero della neoplasia in tutte le sue componenti in atto è l'obiettivo di ogni intervento chirurgico con intenti di radicalità. Va detto che le caratteristiche del distretto stesso rappresentano una limitazione all'applicazione sistematica della chirurgia per le possibili menomazioni funzionali ed estetiche che ne derivano.

La maggior parte dei carcinomi della testa e del collo in stadio iniziale può essere curata sia con intervento chirurgico che con radioterapia, ottenendo percentuali di controllo sovrapponibili per lesioni di pari dimensioni. La scelta della terapia, in questi casi, è influenzata da diversi fattori quali: i risultati estetici e funzionali, le condizioni generali del paziente, la preferenza dello stesso paziente.

In presenza di neoplasie più estese, l'indicazione al trattamento chirurgico diviene preminente per varie ragioni. Innanzitutto si riscontrano percentuali più alte di guarigione locale rispetto a quelle ottenibili con un trattamento radiante, soprattutto nel caso di neoplasie profondamente infiltranti, anche perché i progressi della chirurgia plastica ricostruttiva hanno consentito di ridurre notevolmente le menomazioni estetiche e funzionali conseguenti a interventi chirurgici demolitivi. Il tutto va comunque considerato alla luce di un corretto bilancio tra risultati oncologici e funzionali.

Il concetto di radicalità chirurgica va applicato, laddove possibile, all'intera malattia neoplastica tecnicamente aggredibile e all'unità "tumore primitivo-rete linfatica".

È necessario poter stabilire a priori i margini di resezione con elevata probabilità di cadere in tessuto sano, tenendo conto di alcuni fattori quali l'istologia, la modalità di crescita e di invasione nei tessuti circostanti, la presenza o meno di barriere anatomiche o di vie preferenziali per la diffusione neoplastica, le possibilità ricostruttive e le modalità di trattamento dei campi laterocervicali.

\begin{tabular}{|lcl|}
\multicolumn{1}{|c}{$\begin{array}{c}\text { Terminologia } \\
\text { Robbins }\end{array}$} & $\begin{array}{c}\text { Livello } \\
\text { sottolivello }\end{array}$ & \multicolumn{1}{c|}{ Gruppo } \\
\hline L. sottomentonieri & IA & Sottomentoniero \\
\hline L. sottomandibolari & IB & Sottomandibolare \\
\hline L. giugulari superiori & II & Giugulare superiore \\
\hline L. giugulari medi & III & Giugulare medio \\
\hline L. giugulari inferiori & IV & Giugulare inferiore \\
\hline L. cervicali posteriori & VA & Triangolo posteriore \\
\hline L. sopraclaveari & VB & \\
\hline
\end{tabular}

Tabella II. I livelli di Robbins
Il risultato oncologico va sempre perseguito insieme ad un adeguato risultato funzionale. Ciò presuppone che l'indicazione chirurgica demolitiva venga sempre associata, laddove possibile, a un tempo ricostruttivo.

Le tecniche ricostruttive possono avvalersi di svariate metodiche: lembi di vicinanza, lembi peduncolati o lembi liberi. La scelta fra le varie metodiche dipende dalla sede della lesione primitiva, dall'entità della resezione, dalle condizioni generali e locali del paziente.

\section{Problema linfonodale}

La facilità e precocità di invasione della rete linfatica rende ragione della frequente diffusione regionale per questa via, propria dei tumori maligni epiteliali del distretto cervico-facciale. La frequenza della diffusione linfatica ha comportato nei decenni la messa a punto di procedimenti tecnici e di accorgimenti strategici rivolti a controllarla.

I procedimenti tecnici sono rivolti alla possibilità di ottenere la radicalità chirurgica regionale, più o meno indipendentemente dal tipo e dalla sede del tumore primitivo.

Gli accorgimenti strategici derivano dalla necessità di considerare unitariamente la rilevanza statistica della diffusione linfatica con le relative linee preferenziali di diffusione. A differenza degli interventi sul tumore primitivo, ben diversi da localizzazione a localizzazione, quelli eseguibili sulle aree linfatiche sono ormai standardizzati e riconducibili a pochi modelli, indipendentemente dalla sede del tumore. Possono variare le indicazioni, ma non le tecniche, che vengono qui illustrate.

\section{Tecniche chirurgiche per le aree linfatiche}

\section{Svuotamento radicale tradizionale demolitivo}

Esso comporta, oltre alla rimozione dell'intera rete linfatica di uno dei lati del collo, il sacrificio del muscolo sternocleidomastoideo, della vena giugulare interna e del nervo accessorio spinale. Trova indicazione in presenza di adenopatie di diametro massimo superiore a $3 \mathrm{~cm}$ o fisse o di adenopatie multiple (più di 3 ). In caso di necessità può essere allargato ad altre strutture coinvolte nel processo neoplastico (carotide esterna, nervo ipoglosso, muscoli del piano profondo del collo, cute). Di norma non è eseguibile bilateralmente nella stessa seduta operatoria per le possibili complicanze immediate.

\section{Svuotamento funzionale classico}

Comporta nella sua realizzazione la dissezione e asportazione delle fasce cervicali e del loro contenuto linfonodale, con la conservazione della vena giugulare interna, del muscolo sternocleidomastoideo e del nervo accessorio spinale. Può essere eseguito bilateralmente nella stessa seduta senza 
complicazioni immediate, anche nel caso sia necessario il sacrificio della vena giugulare interna di un lato. Lo svuotamento funzionale classico è indicato in assenza di adenopatie clinicamente apprezzabili o in presenza di adenopatie di dimensioni inferiori a $3 \mathrm{~cm}$, mobili e in genere in numero non superiore a 3 .

\section{Svuotamento selettivo}

A differenza dello svuotamento funzionale classico completo, che prevede la rimozione di tutta la rete linfatica presente nelle varie regioni del collo (livelli dal I al V secondo Robbins, Tabella II), lo svuotamento selettivo comporta la dissezione dei livelli statisticamente a maggior rischio di metastatizzazione linfatica. Per la cavità orale i livelli in questione sono I, II, e III (sopraomoioidei), per la laringe II, III, IV. L'indicazione essenziale per questo tipo di svuotamento è l'assenza di adenopatie palpabili. Come per lo svuotamento funzionale completo, quello selettivo può e deve essere eseguito bilateralmente in caso di localizzazione mediana o paramediana del tumore primitivo.

\section{Indicazioni tattiche}

Gli svuotamenti possono venire eseguiti separatamente o simultaneamente al trattamento del tumore primitivo. Il trattamento separato si ha tipicamente in caso di comparsa di metastasi tardiva in colli inizialmente non trattati, oppure nel caso in cui, per vari motivi, lo svuotamento venga volutamente differito.

Gli svuotamenti simultanei vengono sempre associati agli interventi sul tumore primitivo possibilmente in monoblocco, realizzando così il concetto di simultaneità terapeutica, cronologica e spaziale.

\section{GLI ASPETTI LEGALI}

\section{RISCHI MEDICO-LEGALI IN CASO DI OMISSIONI E DI AZIONI}

\section{Sentenze sull'errore diagnostico}

Per il medico di medicina generale i rischi maggiori sono rappresentati dal danno conseguente al mancato riconoscimento di una condizione clinica richiedente cure indifferibili, al ritardo di diagnosi, alla tardiva proposta di ricovero ospedaliero urgente e al rifiuto di visita medica ambulatoriale o domiciliare, se dovuta (Cassazione penale, sez. VI, sentenza n. 4168/1995).

Presupposto iniziale di una condotta corretta in tutte queste situazioni è il dovere di effettuare un esame fisico del paziente per riconoscere tutti i segni obiettivamente rilevabili di malattia.
Ad esempio il medico che non riconosce i segni e sintomi di un infarto miocardico acuto, ritardando così il necessario ricovero ospedaliero e le cure indifferibili, è responsabile penalmente di omicidio colposo secondo la sentenza n. 113 del 19 gennaio 2004 della Corte di Appello di Milano, II sezione penale.

Il medico non risponde dei danni se l'omessa visita non avrebbe cambiato l'evoluzione clinica del caso (Cassazione civile, sezione III, sentenza n. 19133/2004), ma può comunque rispondere di omissione d'atti d'ufficio se la visita era dovuta (art. 328 del Codice penale).

Il medico che omette di rilevare un nodulo mammario sospetto e di prescrivere con tempestività una mammografia risponde del reato di lesioni colpose in quanto ha omesso indebitamente di prevenire l'aggravarsi di un carcinoma a prognosi infausta, secondo la sentenza del 5 giugno 1989 della Pretura di Ivrea, pubblicata in La Giustizia Penale 1990, II: 682.

\section{II ruolo dell'ASL}

Per la prevenzione delle controversie medico-paziente va rilevato il ruolo non secondario delle organizzazioni sanitarie, in quanto spetta alle ASL:

- la preventiva informazione agli assistiti su quali sono le prestazioni dovute ed esigibili dai medici e quindi su quali sono le aspettative realistiche soddisfabili dai singoli operatori e dal servizio pubblico;

- la gestione delle carenze di personale, servizi e coordinamento che possono aumentare i rischi di errore medico;

- la gestione propositiva degli errori medici, ad esempio sul modello delle "Unità di Gestione del Rischio" attivate al Centro Studi San Raffaele di Milano.

\section{La comunicazione medico-paziente}

Nella pratica il medico tende a gestire in autonomia il confronto e il dissenso, purché non tocchi il suo ruolo. Il vissuto conflittuale dei medici è spesso ispirato alla questione del loro ruolo: quando questo viene messo in discussione da parte di pazienti o di colleghi, si passa frequentemente dalla tendenza all'abituale confronto costruttivo gestito con tolleranza al conflitto ingestibile [4].

Nella percezione della popolazione l'errore medico è dovuto più spesso al sovraccarico di lavoro del personale sanitario, allo scarso tempo dedicato ai pazienti e alla scarsa capacità di comunicazione dei Sanitari [5].

Il gradimento nel rapporto medico-paziente dipende infatti dalla fiducia basata, oltre che sulle capacità tecniche del medico, soprattutto sul giudizio riguardo le sue capacità comunicative di rilevare sia i bisogni che le aspettative personali del paziente [6]. È dimostrato che un medico più em- 
patico e un maggior coinvolgimento del paziente nelle decisioni cliniche rendono il paziente più collaborante e riducono il rischio di contenziosi per responsabilità professionale [7].

\section{I rischi medico-legali di omissioni e di azioni nell'esame fisico}

Le denunce pervenute al tribunale per i Diritti del Malato di Milano nel 2001 in tema di diagnosi hanno segnalato errori medici soprattutto nell'interpretazione di esami diagnostici (53\%), ritardi di diagnosi (22\%) ed errori di diagnosi $(20 \%)$, sebbene in Italia l'80\% delle cause contro i medici si risolvano poi con l'assoluzione degli imputati, dimostrando così l'alta ricorrenza di azioni legali basate su accuse infondate.

Per il medico di medicina generale è possibile ridurre il rischio di contenziosi tenendo conto dei seguenti elementi: il valore riscoperto dell'esame obiettivo, linee guida e limiti normativi, sentenze della giurisprudenza e utilità della comunicazione efficace tra medico e paziente. Un attivo ruolo dell'ASL può ridurre errori medici e contenziosi informando gli utenti sull'appropriatezza nell'uso dei servizi e organizzando le Unità di Gestione del Rischio (UGR) per aiutare i Sanitari ad imparare dall'errore.

\section{L'esame obiettivo}

L'importanza di effettuare un accurato esame obiettivo del paziente è dimostrata da un recente studio svolto dall'equipe del dottor Brendan Reilly al Cook Country Hospital di Chicago secondo cui un'accurato rilievo dei segni fisici porta a modificare il sospetto diagnostico iniziale e il successivo iter gestionale diagnostico-terapeutico in un paziente su quattro [8]. Si dimostra così l'utilità di un uso maggiore della semeiotica fisica al fine di una più corretta diagnosi e di un ricorso più mirato $\mathrm{e}$ appropriato alla diagnostica strumentale.

Un esempio di esame obiettivo a rischio legale è rappresentato dall'esplorazione rettale e vaginale.

Secondo linee guida per l'iter diagnostico del dolore ai quadranti inferiori dell'addome, è necessario ed appropriato l'esame obiettivo mediante l'esplorazione rettale per il dolore in fossa iliaca destra o sinistra e mediante l'esplorazione vaginale per il dolore pelvico, a uno dei tre quadranti inferiori addominali e in particolare a quello ipogastrico [9]. Le esplorazioni sono indicate anche in caso di disturbi persistenti delle basse vie urinarie [10] e di disturbi ano-rettali [11]. Indipendentemente dai sintomi, l'esplorazione rettale fa parte dello screening del cancro colon-rettale [12]. Un accurato inquadramento clinico del paziente può consentire infatti di rilevare neoformazioni patologiche, stabilire quali sono gli accertamenti diagnostici più mirati al problema, con quale grado di urgenza devono essere richiesti, chi è lo specialista più indicato a cui indirizzare il paziente: chirurgo, gastroenterologo, ginecologo, proctologo, urologo o anche psichiatra nel caso di disturbo da conversione o da somatizzazione in assenza di rilievi fisici e strumentali patologici. Nel corso dell'istruzione universitaria gli studenti vengono raramente educati sulle regole etiche che dovrebbero guidare l'esecuzione di procedure delicate come l'esplorazione rettale e l'esplorazione vaginale [13]. Esplicitare preventivamente con chiarezza la motivazione e la procedura di questi accertamenti fisici è importante, particolarmente nei soggetti psichiatrici in cui accertare l'acquisizione di un valido consenso può essere difficile [14] e nei pazienti di sesso diverso rispetto a quello del medico poiché qualsiasi contatto fisico non consentito e giustificato da una finalità diagnostica può essere interpretato come violenza sessuale ai sensi della Legge n. 66 del 15.02.1996 [15,16].

Al contrario l'omissione di visita potrebbe causare un ritardo colposo di diagnosi in caso di cancro rettale, prostatico o ovarico [17].

\section{Linee guida e limiti normativi}

Il medico, ai sensi dell'art. 31 del DPR 270/2000, ha compiti di diagnosi e cura verso gli assistiti, ma ai sensi dell'art. 15-bis dello stesso decreto deve anche assicurare l'appropriatezza nell'utilizzo delle risorse sanitarie per l'erogazione dei Livelli Essenziali di Assistenza (LEA) riducendo il ricorso ad esami ingiustificati mediante adozione di principi della Medicina Basata sull'Evidenza (EBM).

Ladozione di linee guida diagnostiche condivise e il confronto tra gruppi di colleghi nell'ambito delle cure primarie può ridurre la tendenza documentata a richiedere più test diagnostici del necessario [18]. Questa tendenza è spesso favorita da richieste improprie degli stessi pazienti [19] o dal timore dei medici di trascurare ipotesi diagnostiche incorrendo in responsabilità professionali [20].

Ma in medicina questa tendenza non riduce necessariamente il rischio di ritardi nelle diagnosi, a causa di lunghe liste d'attesa per l'esecuzione degli esami strumentali, necessità di accertamenti successivi richiedenti tempi d'attesa ulteriori, incomprensione o scarsa adesione dei pazienti alle prescrizioni [21], errori nell'interpretazione dei risultati e falsi negativi nell'esito degli esami, che danno l'infondata sicurezza di poter escludere malattie confidando incautamente nel solo accertamento strumentale. Ad esempio, un'ecografia prostatica può non rilevare la presenza di un cancro che appare come una neoformazione isoecogena e così indurre a sospendere ulteriori accertamenti invece necessari.

Tuttavia la Cassazione penale con sentenza $n$. 9279/2003 ha affermato che "il dubbio diagnosti- 
co deve indurre ad utilizzare gli strumenti diagnostici necessari per risolverlo e la gravità della patologia o delle conseguenze del suo mancato accertamento non può che accentuare questo obbligo estendendolo anche alla necessità di indagare sulle ipotesi patologiche più remote poiché il medico che venga meno a questi obblighi non può non essere considerato in colpa".

\section{DISCUSSIONE MEDICO-LEGALE DEL CASO IN OGGETTO}

Nel febbraio 2006, il tribunale di Cagliari condannava gli imputati (il medico esecutore, l'anestesista e l'infermiera professionale) per lesioni colpose gravi ai sensi dell'art. 590 del Codice penale, a causa di negligenza, imprudenza ed imperizia medica. Siamo a tutt'oggi in attesa del secondo grado di giudizio.

Il nervo accessorio spinale rappresenta l' $11^{\circ}$ paio dei 12 nervi cranici: è un nervo esclusivamente motore e nasce dal corno anteriore dei primi cinque o sei segmenti del midollo cervicale, ha un decorso in parte intracranico e in parte extracranico, dal quale esce per innervare, in regione laterocervicale, il muscolo sternocleidomastoideo e in regione dorsale alta per innervare la porzione superiore del muscolo trapezio.

Lattuale indagine di consulenza medico-legale ha evidenziato gli esiti, ormai consolidati, certamente legati alla biopsia linfonodale eseguita in data 28 aprile. Tale consulenza non ha la pretesa né lo scopo di considerare e valutare aspetti quali la colpa, la negligenza e l'imperizia medica, ma la causa è senza dubbio da ascrivere alla biopsia linfonodale laterocervicale destra, perché tale patologia non riconosce che cause traumatiche in soggetti perfettamente sani a causa di traumi violenti o rotture per tagli netti [1].

Non esistono, in letteratura, rotture, parziali e/o totali, dei nervi, se non in concomitanza e a causa di molteplici altre malattie.

La biopsia linfonodale del triangolo posteriore del collo è una pratica routinaria se viene eseguita correttamente, ma può nascondere qualche insidia per chi vi si approccia con troppa disinvoltura o superficialità.

Infatti, l'escissione completa del linfonodo, che oggi viene soppiantata dall'agoaspirato proprio per evitare danneggiamenti nervosi, viene riservata ai casi dubbi.

Dovrebbe essere eseguita "a cielo aperto" ovvero in sala operatoria, con blanda anestesia senza l'uso del curaro e con le precauzioni che seguono: l'incisione viene sempre fatta dall'alto verso il basso e mai trasversalmente al decorso del nervo.

Il fascio nervoso (perché sono molti i nervi che passano in quella zona) deve essere isolato, nervo per nervo, e solo allora, sicuri di non commettere danneggiamenti nervosi, si asporta il linfonodo da esaminare.
Per chi non vuole attuare questa pratica, che comunque risulta essere la più sicura, si può biopsiare a "cielo chiuso" facendo, però, uso dell'ecografo che aiuta il medico a trovare il nervo e il suo esatto decorso.

Il taglio deve essere longitudinale al decorso nervoso, perché si ha bisogno di poter divaricare una ampia zona di campo operatorio e quindi "ben esposto".

Nello specifico caso di A.M., il medico doveva sapere che la lesione del nervo accessorio spinale è la complicanza più frequente in caso di procedure chirurgiche che interessano il triangolo posteriore del collo.

Il tragitto di tale nervo è superficiale, tanto che passa sopra ai linfonodi, solo un breve tratto è intracranico.

Ma non esiste, comunque, alcuna scusante: il decorso del nervo, sia esso superficiale o profondo, va conosciuto e l'escissione del linfonodo satellite va saputa fare: numerosissima è la letteratura, soprattutto in ambito statunitense, che riporta casi di malpractice medica relativi a rotture nervose imputabili alla mancata conoscenza dell'anatomia del triangolo posteriore del collo.

A questo punto, corre l'obbligo di formulare alcune domande relative al caso in oggetto:

- perché non è stata effettuata la biopsia a cielo aperto, considerate le insidie di tale procedura oggi routinaria?

- perché il taglio di approccio alla cute è ortogonale al decorso del nervo e non longitudinale (contrariamente a quanto è stato fatto nella biopsia successiva che è stata effettuata correttamente), quando quest'ultimo espone maggiormente il campo d'azione?

- come mai il Collega non ha sottoposto il paziente, dopo alcuni giorni dalla biopsia, ad alcune banali prove di funzionalità del muscolo trapezio e della spalla?

- può, il medesimo Sanitario, conoscere solo a grandi linee cosa è, come è fatto e quali limiti anatomici possiede il cosiddetto "punto di Erb", zona anatomica presente nel triangolo posteriore del collo che al contrario deve essere conosciuto come la Bibbia da quei chirurghi che operano nella zona laterale del collo proprio per non incorrere nella rottura di qualche elemento nervoso? È come se chi opera sulla tiroide non isolasse i due nervi ricorrenti che ci danno la voce e la giusta respirazione.

Il trauma è avvenuto su persona perfettamente integra in ogni suo distretto corporeo, interrompendo una continua attività sportiva, ora negata, e lavorativa nella quale l'uso delle mani, soprattutto quella dominante, risultava determinante nell'integrità anche psichica dell'individuo.

Alla domanda, quindi, se il nervo accessorio spinale destro del Signor A. M. fosse integro e perfettamente efficiente prima del trauma del 28 aprile 2003, la risposta è, senza alcun dubbio, sì, 
e la negazione di ciò rimane nel mero campo delle illazioni ed è destinata a cadere in assenza di valida dimostrazione.

Siamo di fronte agli esiti di una lesione gravissima che comporta di sicuro l'esclusione dalla attività esercitata fino al danno ed anche dalla "esclusione sociale" del paziente da attività sportive fino ad allora assiduamente praticate.

Prima delll'intervento A. M., pur non lavorando regolarmente, esercitava il tennis a livello agonistico ed era stato segnalato per effettuare un corso per diventare istruttore, attività resa ormai impossibile dalla tipologia di danno subito. Certamente, infatti, non potrà compiere in futuro attività lavo- rative per il cui svolgimento risulti essenziale tutto l'arto destro (ha, attualmente, già problemi per l'uso del computer e della penna, tanto per fare un esempio).

Da non sottovalutare, peraltro, il danno psicologico subito che va ad aggiungersi a quello già presente a causa dell'emopatia linfatica e che comporterà, sicuramente, una continua sorveglianza terapeutica e un sostegno psicologico di supporto.

Per quanto sopra, i postumi residui del danno subito in data 28 aprile 2003 costituiscono danno grave alla persona, identificabile come danno biologico, in riferimento alla riduzione della integrità psicofisica in sé.

\section{BIBLIOGRAFIA}

1. Ewing MR, Martin H. Disability following radical neck dissection: an assessment based on the postoperative evaluation of 100 patients. Cancer 1952; 5: 873-83

2. Tomasello G, Iadicola A, Mazzotta MP, De Luca B, Di Chiara C, Arsena P. Studio sulle neoformazioni del collo e del torace. Università degli studi di Palermo, 2004

3. Messiaen T, Lefebvre C, Van Beers B, Sempoux C, Cosyns JP, Geubel A. Hepatic angiomyo(myelo)lipoma: difficulties in radiological diagnosis and interest of fine needle aspiration biopsy. Liver 1996; 16: 338-41

4. Ricerca e Pratica 2002; 18: 6

5. Blendon RJ, DesRoches CM, Brodie M et al. Patient safety: views of practicing physicians and the public on medical errors. $N$ Eng J Med 2002; 347: 1933 e 1965

6. Hantho A, Jensen L, Malterud K. Mutual understanding: a communication model for general practice. Scand J Prim Health Care 2002; 20: 244-51

7. Flaherty M. Good communication cuts risk. Physician's Financial News 2002; 20: S10-S11

8. Reilly BM. Physical examination in the care of medical inpatients: an observational study. The Lancet 2003; 362: 1100-5

9. Healey PM. Il processo decisionale nella diagnosi medica. Il Pensiero Scientifico: Roma, 1992, pagg. 58-61

10. AUA Practice Guidelines Committee. AUA guideline on management of benign prostatic hyperplasia (2003). Chapter 1: Diagnosis and treatment recommendations. J Urol 2003; 170: 530-47

11. Gatti G. Dal Sintomo, alla diagnosi, alla terapia. Minerva Medica: Torino, 2000, pag. 393

12. Doria-Rose VP, Levin TR, Selby JV, Newcomb PA, Richert-Boe KE, Weiss NS. The incidence of colorectal cancer following a negative screening sigmoidoscopy: implications for screening interval. Gastroenterology 2004; 127: 989-90

13. Singer PA. Intimate examinations and other ethical challenges in medical education. BMJ 2003; 326: 62-3

14. Stagno SJ. Agich GJ. Consent in patients with mental illness. History and philosophy. Current Opinion in Psychiatry 1997; 10: 423-6

15. Cass. Pen. III, 23 luglio 1994, in Zacchia 357, 1996

16. Cass. Pen. III, 23 marzo 1986, in Cass. Pen. 715, 1987

17. Goff BA, Mandel LS, Melancon CH, Muntz HG. Frequency of symptoms of ovarian cancer in women presenting to primary care clinics. JAMA 2004; 291: 2705-12

18. Verstappen WH, van der Weijden T, Sijbrandij J et al. Effect of a practice-based strategy on test ordering performance of primary care physicians: a randomized trial. JAMA 2003; 289: 2407

19. Ricerca e Pratica 2003; 19: 235-243

20. Little P, Dorward M, Warner G et al. Importance of patient pressure and perceived pressure and perceived medical need for investigations, referral, and prescribing in primary care: nested observational study. BMJ 2004; 328: 444-6

21. Dowell J, Jones A, Snadden D. Exploring medication use to seek concordance with "non-adherent" patients: a qualitative study. Br J Gen Pract 2002; 52: 24-32 